\title{
INFLUENCE OF KERF WIDTHS TO AN ASSIST GAS FLOW IN LASER CUTTING
}

\author{
${ }^{* 1}$ Toshihide Hanari, ${ }^{* 1}$ Toshiharu Muramatsu, ${ }^{* 2}$ Masanori Yamashita, ${ }^{* 2}$ Jun Sakakibara \\ ${ }^{* 1}$ Applied Laser Technology Development Office, Applied Laser Technology Institute, Japan Atomic Energy Agency \\ 65-20 Kizaki, Tsuruga, Fukui, 914-8585, Japan \\ hanari.toshihide@jaea.go.jp
}

${ }^{* 2}$ Department of Engineering Mechanics and Energy, University of Tsukuba 1-1-1 Tennodai, Tsukuba, Ibaraki, 305-8577, Japan

\begin{abstract}
Laser cutting technology has a characteristic of generating low waste volume, and is expected to be applied to reactor decommissioning. Ejection of molten metal from deep in a kerf has been a challenge for the technique. As a kerf is narrow, an assist gas flow decays with increasing kerf depth. Therefore thermohydraulic interaction between the assist gas and the molten metal plays an important role in kerf formation. In order to quantify the influence of the assist gas flow to the kerf width, we evaluated several parameters such as kerf width, working distance and flow rate. Flow field of the assist gas in a mock-up is investigated using PIV. Pressure loss of the assist gas flow decreased with increasing kerf width, and so the assist gas became easier to reach deep in the kerf. This indicated that kerf width is an important parameter in the laser cutting process.
\end{abstract}

\section{INTRODUCTION}

Laser cutting is a thermal cutting technique that involves the melting of a metal plate through laser irradiation and detaching it from a cutting kerf using an assist gas flow [13]. Figure 1 shows a schematic of a laser cutting process. The material is firstly irradiated and heated up by a laser beam. When the material temperature reaches its melting point, the material phase changes from solid to liquid. An assist gas flow is applied to the molten metal and removes it along the kerf front.

Laser cutting is well-known for its low waste volume generated during the cutting process and is expected to be applied to reactor decommissioning towards a thickness of over $150 \mathrm{~mm}$. Laser cutting experiments of thick steel plates are conducted using a $10 \mathrm{~kW}$ fiber laser system. However, laser cutting of over $100 \mathrm{~mm}$ thick steel plate has not been achieved yet. This is due to the ejection of molten metal from the kerf to the other side of the steel plate. As the cutting kerf is narrow, the assist gas flow weakens with increasing kerf depth. This reduces the shear and pressure force to the molten metal in the deep part of the kerf. Therefore thermohydraulic interaction between the assist gas and the molten metal plays an important role in the formation of a kerf. Deep inside the kerf of a thick plate, the hydrodynamic characteristics of molten metal are determined by physical properties such as viscosity and surface tension. However, the ejection mechanism of the molten metal has not been clarified due to the difficulty in thermofluid measurement in laser cutting. Thus, we investigate the hydrodynamic characteristics of the assist gas flow in a kerf using a mock-up that simulates the laser cutting kerf.

In the present paper, we focus on the effect on the hydrodynamic characteristics to the laser cutting performance by an assist gas flow, and investigate the assist gas flow field in a mock-up using particle image velocimetry (PIV). In order to quantify the influence of the assist gas flow, we evaluate several parameters such as kerf width, working distance, and flow rate of the assist gas.

\section{EXPERIMENTAL SETUP}

Figure 2 shows the mock-up for the PIV measurement. The experiment was conducted in a test section which imitates a laser cutting kerf and a straight nozzle. An assist gas flow was jetted from the nozzle towards the test section.

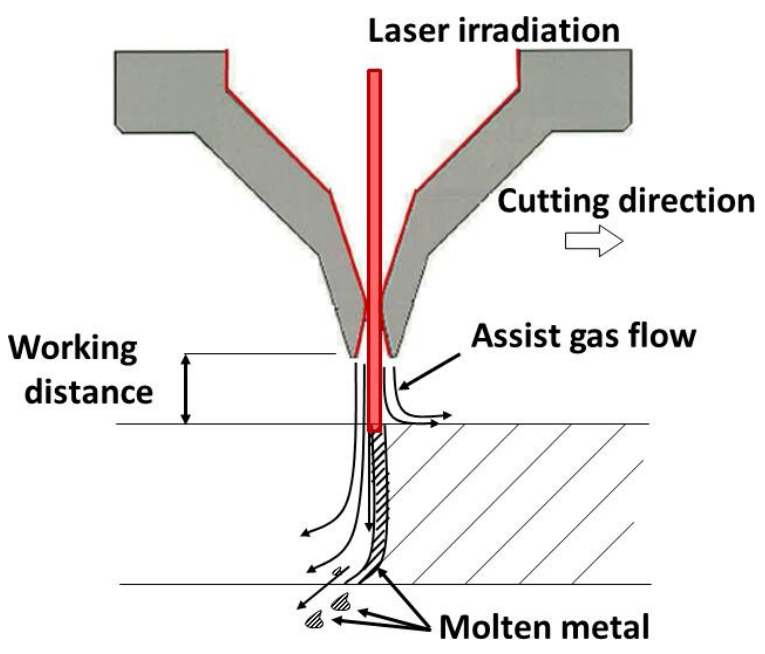

Fig. 1 Laser cutting process 


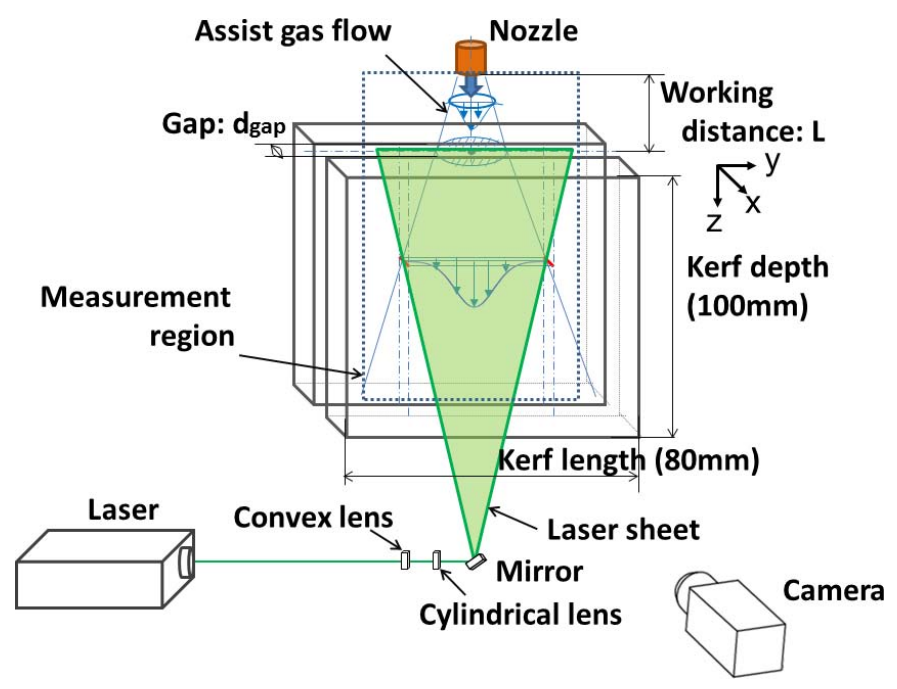

Fig. 2 Experimental apparatus

The test section was made from plexiglass of $80 \mathrm{~mm}$ in length and $100 \mathrm{~mm}$ in depth. The kerf, which was represented by the gap between the test section walls, was set at 1,2 , and $4 \mathrm{~mm}\left(d_{\text {gap }}\right)$. The diameter of the nozzle was $3 \mathrm{~mm}$. Compressed air was chosen as the assist gas, and the flow rate $Q$ was set to 11 and $21 \mathrm{~L} / \mathrm{min}$. This corresponds to a Reynolds number of 5000 and 10000 , respectively. The working distance $(L)$ between the test section and the nozzle was 2,5 and $10 \mathrm{~mm}$. Table 1 lists the six cases to be conducted in this study.

Table. 1 Experimental conditions

\begin{tabular}{c|ccc}
\hline Case & $d_{\text {gap }}[\mathrm{mm}]$ & $L[\mathrm{~mm}]$ & $R e$ \\
\hline A & 1 & 10 & 5000 \\
B & 2 & 10 & 5000 \\
C & 4 & 10 & 5000 \\
D & 2 & 2 & 5000 \\
E & 2 & 5 & 5000 \\
F & 2 & 10 & 10000 \\
\hline
\end{tabular}

In order to measure the velocity field, PIV was applied. The assist gas was homogeneously seeded with olive oil mist using a particle generator (TSI, 6-Jet Atomizer Model 9306A). For the PIV measurement, a laser light sheet of 1 mm thick was produced by a double-pulse Nd:YAG laser (Litron Lasers, Nano S 50-20PIV, $50 \mathrm{~mJ} /$ pulse, $20 \mathrm{~Hz}$ ) through a cylindrical lens to illuminate the flow fields in the test section. A CMOS camera (Photron, Fastcam SA4, 1024 pixels $\times 1024$ pixels) was positioned normally to the sheet to view the tracer particles at a measurement region of interest. The captured images were stored in the camera's memory. The laser and the camera were synchronized with a digital delay generator (Quantum composers, Model 9618). Our PIV system could typically capture 2500 successive timedependent pairs of images. Since a velocity field was calculated from captured double images, 1250 instantaneous two-dimension velocity were obtained for each case.

The time intervals between double pulses for $\mathrm{Re}=5000$ and 10000 were $30 \mathrm{~ms}$ and $15 \mathrm{~ms}$, respectively, with a sampling period was $0.1 \mathrm{~s}$. The field of view of the camera was approximately $110 \mathrm{~mm}$ by $110 \mathrm{~mm}$. The measured displacement error of the tracer particles was 0.1 pixels, which led to the errors of the instantaneous velocity of approximately $3.4 \mathrm{~m} / \mathrm{s}$ and $6.8 \mathrm{~m} / \mathrm{s}$ for $\mathrm{Re}=5000$ and 10000 , respectively. The PIV interrogation was performed by our in-house software. Details of the algorithm were described in a previous paper [4].

\section{RESULTS AND DISCUSSION}

\subsection{Mean center velocity of the assist gas flow}

For each case, a mean velocity profile was obtained by averaging 1250 instantaneous velocity maps. The mean center velocity, $U_{z}$, of the assist gas flow for different kerf widths (Cases A, B and C) are shown in Fig. 3. The surface of the test section was defined as $z=0 \mathrm{~mm}$. The velocity was normalized by the free jet center velocity at $\mathrm{z}=10 \mathrm{~mm} . \Delta U$ was velocity difference between $U_{z}$ at $z=10 \mathrm{~mm}$ and the free jet center velocity at the same depth. Thus, the normalized velocity at $z=10 \mathrm{~mm}$ was equal to 1 . Compared with the case of the free jet, the velocity was lower for the case with $1 \mathrm{~mm}$ kerf width. The decrease could be caused by the friction of the walls. Velocities for the case of 2 and 4 mm kerf width were close to those of the free jet case. This suggested the loss due to the wall friction was minimal when the kerf was wide enough. Since diffusing momentum of the jet was affected by the wall of the test section, we considered that the center velocity in the test section is maintained.

Figure 4 shows the mean center velocity of the assist gas flow with different working distances (Cases B, D and E). The center velocity of Case B at $z=10 \mathrm{~mm}$ was chosen as the reference for subtraction and normalization this time. The velocity profiles of the test section were similar for all cases. We regard that the velocity is independent of the working distance, given that the working distance of the assist gas nozzle is shorter than potential core length of the assist gas flow.

Figure 5 shows the relationship between the mean center velocity of the assist gas flow and the Reynolds number (Cases B and F). Again, the center velocity of Case B at $z=$ $10 \mathrm{~mm}$ was chosen as the reference. Similar to the previous comparison of working distances, the velocity difference between the two cases was insignificant. This suggests the normalized velocity is insensitive to the Reynolds number. 


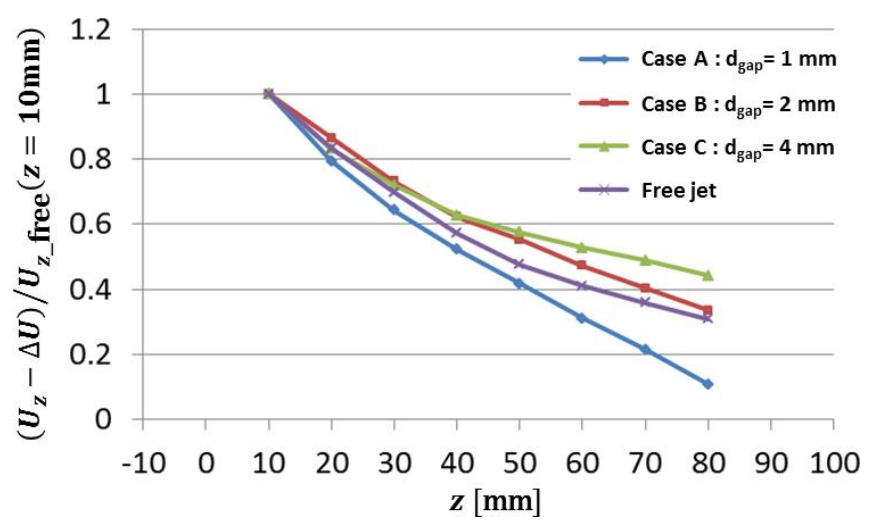

Fig. 3 Normalized mean center velocity of the gas flow with different kerf widths (Cases A, B and C)

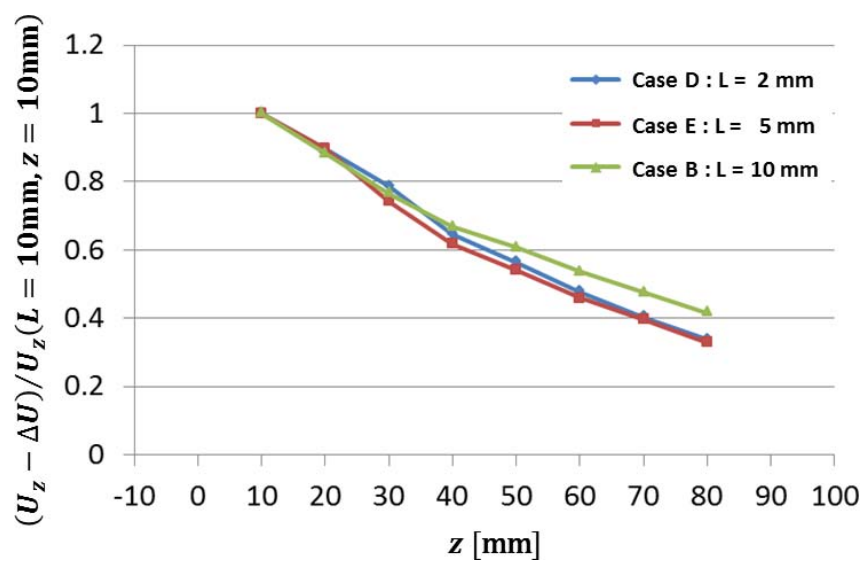

Fig. 4 Normalized mean center velocity of the gas flow with different working distances (Cases B, D and E)

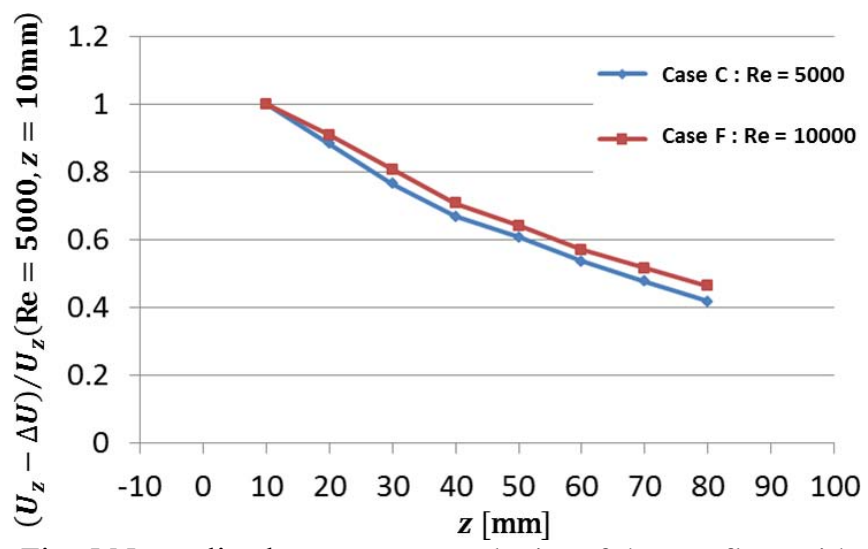

Fig. 5 Normalized mean center velocity of the gas flow with different Reynolds numbers (Cases B and F)

The above results show that the kerf width has a much stronger influence on the center velocity in the test section than those of the working distance and the Reynolds number. When the kerf becomes narrower, the friction contributed by the test section wall increases and the velocity of the assist gas flow decreases. As a result, the momentum of the flow that exerts on the molten metal in the kerf becomes weaker which leads to inefficient ejection. In the next section, we will evaluate the pressure loss of the gas flow caused by the wall friction, the section inlet/outlet.

\subsection{Pressure loss estimation}

We estimated the pressure loss in term of the energy loss in the test section. The total pressure loss $\Delta P$ might be expressed as

$$
\Delta P=\Delta P_{\text {in }}+\Delta P_{\text {wall }}+\Delta P_{\text {out }},
$$

with

$$
\begin{aligned}
& \Delta P_{\text {in }}=\zeta_{\text {in }} \frac{\rho U_{\text {in }}^{2}}{2}, \\
& \Delta P_{\text {wall }}=\zeta_{\text {wall }} \frac{\rho U_{\text {wall }}^{2}}{2}, \\
& \Delta P_{\text {out }}=\zeta_{\text {out }} \frac{\rho U_{\text {out }}^{2}}{2} .
\end{aligned}
$$

$\Delta P, \zeta, \rho$, and $U$ represent the pressure loss, the loss coefficient, the viscosity and the center velocity, respectively. The subscripts in, wall and out represent inlet, wall friction and outlet, respectively. The inlet and outlet velocity were estimated by extrapolating the measured center velocity. The velocity $U_{\text {wall }}$ was calculated from the hydraulic diameter and the flow rate was estimated from the velocity of assist gas flow near the center.

Figure 6 shows the estimated pressure loss for different kerf widths (Cases A, B and C) at a test section depth of 100 $\mathrm{mm}$. The pressure loss is lower for wider kerfs. Compared with the pressure loss for a $1 \mathrm{~mm}$ kerf, the total loss is reduced by approximately $60 \%$ and $80 \%$ for the case of the $2 \mathrm{~mm}$ and $4 \mathrm{~mm}$ kerf, respectively. The loss due to the wall friction is significantly decreased by $90 \%$ for the case of the $2 \mathrm{~mm}$. In other word, the assist gas flow becomes easier to reach deep in the kerf to blow away the molten metal. Hence, it is possible to improve the performance of laser cutting by increasing the kerf width.

Figure 7 shows a similar analysis of the pressure loss at a test section depth of $150 \mathrm{~mm}$, with the outlet velocity estimated by extrapolating the measured center velocity at a test section depth of $100 \mathrm{~mm}$. As the depth increased from $100 \mathrm{~mm}$ to $150 \mathrm{~mm}$, the loss due to the wall friction increased, but the outlet loss decreased. The increase of the wall friction loss confirms the decrease of the assist gas momentum for the ejection of molten metal in the deep part of the kerf.

Figure 8 shows the estimated pressure loss for different working distances (Cases B, D and E). There is no significant difference among the cases. Therefore, it can be concluded that the total pressure loss is independent of the working distance shorter than $10 \mathrm{~mm}$.

The estimated pressure loss for different Reynolds numbers (Cases B and F) is shown in Fig. 9. Compared to the results for the case of $\mathrm{Re}=5000$, the pressure loss at $\mathrm{Re}=10000$ increases approximately 7.2 times, 3.6 times and 3.7 times for the inlet, the wall friction, and the outlet, respectively. The increase of inlet loss has limited the flow of the assist gas into the kerf. Also, the decrease of velocity of the assist 


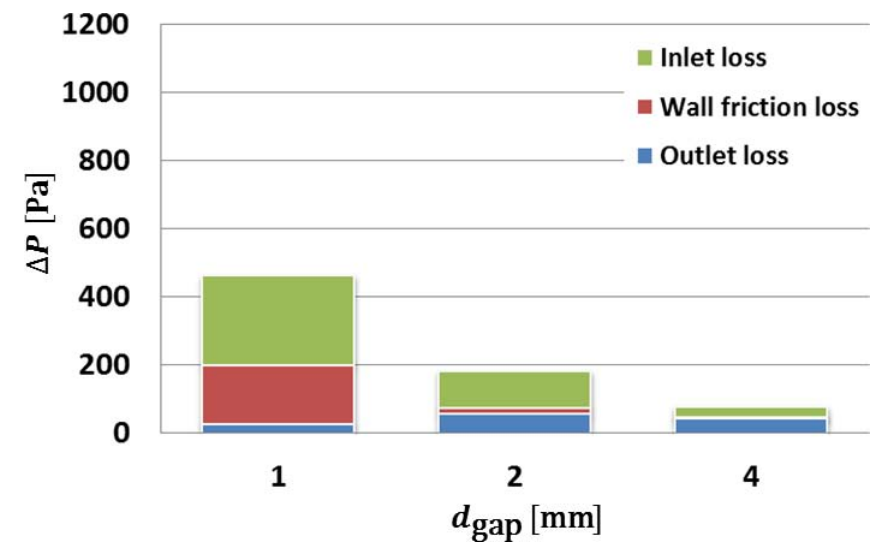

Fig. 6 Pressure loss of different kerf widths (Cases A, B and C) at kerf depth of $100 \mathrm{~mm}$

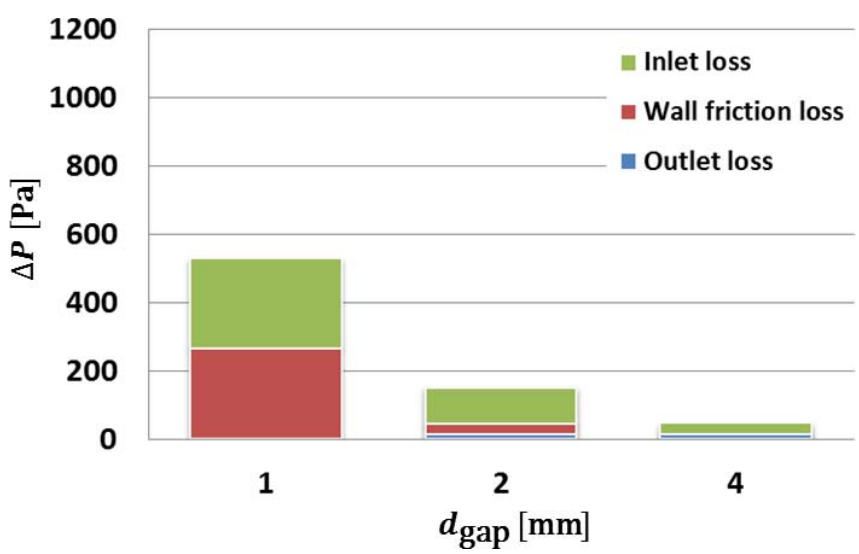

Fig. 7 Pressure loss of different kerf widths (Cases A, B and C) at a kerf depth of $150 \mathrm{~mm}$

gas flow in the kerf is larger with increasing the wall friction loss.

From the results, it can be concluded that the increase of the kerf width from $1 \mathrm{~mm}$ to $2 \mathrm{~mm}$ results in a reduction of approximately $60 \%$ in the total pressure loss. The reduction is mainly due to wall friction. Under this condition, the assist gas flow becomes easier to reach deep in the kerf, and the momentum can help the ejection of molten metals. This is an effective method to improve the laser cutting performance. Moreover, when the kerf depth increases from $100 \mathrm{~mm}$ to $150 \mathrm{~mm}$, the loss due to wall friction increases. This could explain the decrease of momentum of the assist gas flow for the ejection of molten metal in the deep part of the kerf. However, a larger kerf width may possibly improve the laser cutting performance when compared with a narrow kerf.

On the other hand, the working distance from $2 \mathrm{~mm}$ to $10 \mathrm{~mm}$ doesn't have any significant impact on the pressure loss as compared with that of the kerf width. Also, the increase of Reynolds number causes an increase of the pressure loss. In particular the increase of the inlet loss limits an inflow of the assist gas into the kerf. However, this can be compensated by a wider kerf.

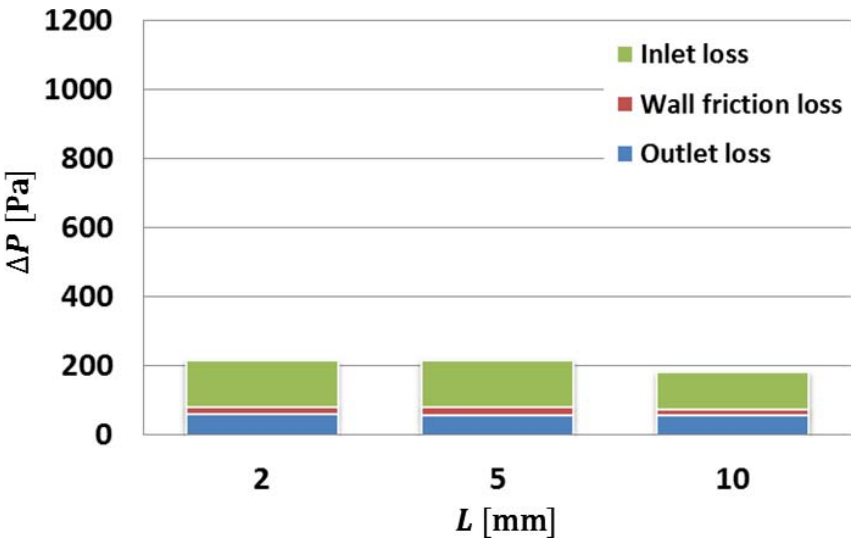

Fig. 8 Pressure loss of different working distances (Cases B, D and E)

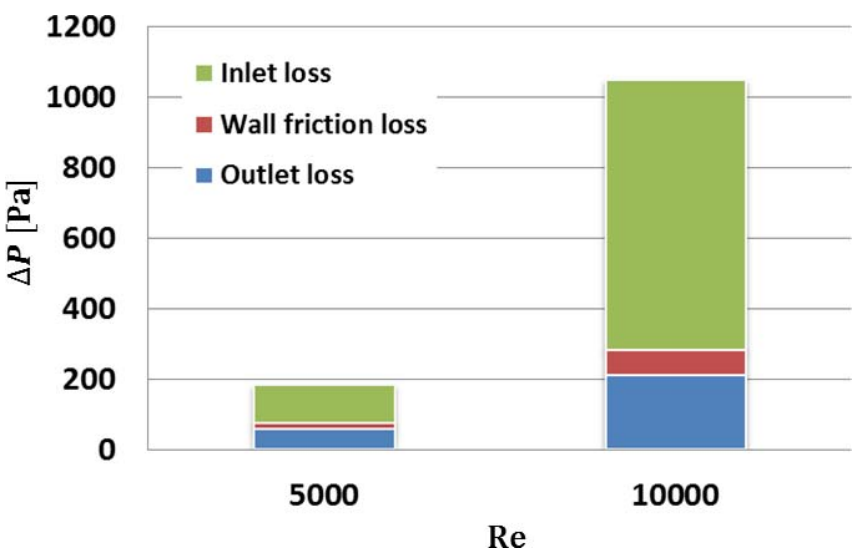

Fig. 9 Pressure loss of different Reynolds numbers (Cases B and F)

\section{CONCLUSION}

We measured the assist gas flow field with PIV in a mock-up laser cutting kerf to evaluate the influences of kerf width, working distance and Reynolds number. The pressure loss of the assist gas flow decreases with increasing kerf width, and so it becomes easier for the assist gas flow to reach deep in the kerf. This suggests a way to improve the performance of the laser cutting. On the other hand, pressure loss due to the wall friction increases with the kerf depth, so the ejection of molten metals becomes inefficient as the momentum of assist gas flow is weaker. However, a wider kerf may imply a possibility to compensate this momentum loss in a deep part of the kerf.

In our study, the pressure loss doesn't show any dependency with the working distance. A larger Reynolds number causes an increase of the pressure loss. Specifically, the increase of the inlet loss reduces the gas flow into the kerf.

We conclude that the kerf width is an important parameter in the laser cutting process. The hydrodynamic parameters such as the assist gas flow rate and the pressure loss strongly affect the laser cutting performance. 


\section{REFERENCES}

[1] Wandera C., Salminen A., Kujanpaa V., Inert gas cutting of thick-section stainless steel and medium-section aluminum using a high power fiber laser, J. Laser Appl., 21, pp. 154-161 (2009).

[2] Kovalev O.B., Yudin P.V., Zaitsev A.V., Modeling of flow separation of assist gas as applied to laser cutting of thick sheet metal, Appl. Math. Model., 33, pp. 37303745 (2009).

[3] Hirano K., Fabbro R., Experimental investigation of hydrodynamics of melt layer during laser cutting of steel, J. Phys. D: Appl. Phys., 44, 105502 (2011).

[4] Sakakibara J., Nakagawa M., Yoshida M., Stereo-PIV study of flow around a maneuvering fish, Exp. Fluids, 36, pp. 282-293 (2004). 